\title{
Magnetic field of a ribbon busbar of finite length
}

\author{
Dariusz Kusiak ${ }^{1, *}$, Tomasz Szczegielniak ${ }^{2}$, and Zygmunt Piątek ${ }^{3}$ \\ ${ }^{1}$ Department of Electrotechnics, Czestochowa University of Technology, Częstochowa, Poland \\ ${ }^{2}$ Institute of Environmental Engineering, Czestochowa University of Technology, Częstochowa, Poland \\ ${ }^{3}$ Professor emeritus, Czestochowa University of Technology, Częstochowa, Poland
}

\begin{abstract}
Using the analytic method based on the Biot-Savart law for the electromagnetic field, the distribution of the magnetic field of a ribbon busbar of finite length has been determined. The Mathematica program was used to visualize the solutions obtained. This allowed quick field analysis after changes of geometrical or electrical parameters of systems under examination.
\end{abstract}

\section{Introduction}

In case of a ribbon busbar of length $l$ (Fig. 1), i.e. the conductor of rectangular section for which $b>>a$, the magnetic field generated by the direct current or slow alternating sinusoidal current $\underline{I}$ at any point $X(x, y, z)$ is determined much easier than in case of rectangular bus $[1,2]$, because the result is obtained after single integration [1].

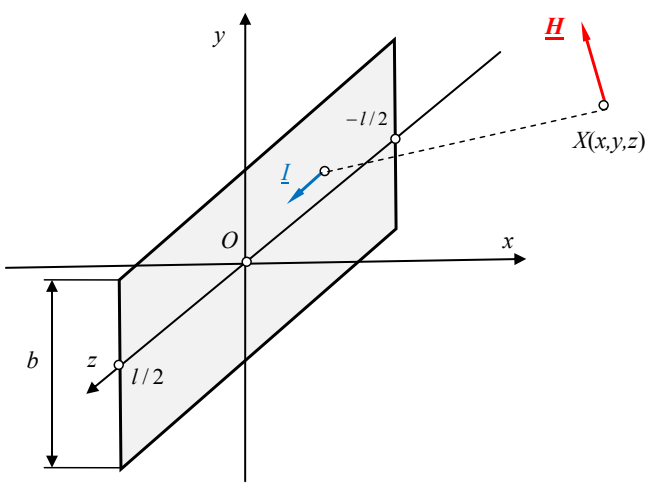

Fig. 1. Ribbon busbar of finite length.

However, if we already have solutions of studies $[1,3]$ at our disposal, then we can determine the magnetic field components through determination of limits of these functions when the crosswise dimension of the busduct is $a \rightarrow 0$. Then, the magnetic field component along the axis $O x$ is as follows

$$
\begin{aligned}
& \underline{H}_{x}^{(t)}(x, y, z)=\operatorname{Limit}_{a \rightarrow 0} \underline{H}_{x}(x, y, z)= \\
& \frac{I}{8 \pi b}\left\{\begin{array}{l}
2 \ln \frac{2 z-l+F_{1}(x, y, z)}{2 z-l+F_{2}(x, y, z)}+ \\
\ln \frac{F_{2}(x, y, z)(l-2 z)+8 l z+(l+2 z) F_{3}(x, y, z)}{F_{1}(x, y, z)(l-2 z)+8 l z+(l+2 z) F_{4}(x, y, z)}+ \\
\ln \frac{-F_{2}(x, y, z)(l-2 z)+8 l z+(l+2 z) F_{3}(x, y, z)}{-F_{1}(x, y, z)(l-2 z)+8 l z+(l+2 z) F_{4}(x, y, z)}
\end{array}\right\}
\end{aligned}
$$

and the magnetic field component along the axis $O y$ is as follows

$$
\begin{aligned}
& \underline{H}_{y}^{(t)}(x, y, z)=\operatorname{Limit}_{a \rightarrow 0} \underline{H}_{y}(x, y, z)= \\
& \frac{I}{4 \pi b}\left\{\begin{array}{l}
\operatorname{arctg} \frac{(b-2 y)(l-2 z)}{2 x F_{1}(x, y, z)}+\operatorname{arctg} \frac{(b+2 y)(l-2 z)}{2 x F_{2}(x, y, z)}+ \\
\operatorname{arctg} \frac{(b-2 y)(l+2 z)}{2 x F_{4}(x, y, z)}+\operatorname{arctg} \frac{(b+2 y)(l+2 z)}{2 x F_{3}(x, y, z)}
\end{array}\right\}
\end{aligned}
$$

where:

$$
\begin{aligned}
& F_{1}(x, y, z)=\sqrt{4 x^{2}+(b-2 y)^{2}+(l-2 z)^{2}}, \\
& F_{2}(x, y, z)=\sqrt{4 x^{2}+(b+2 y)^{2}+(l-2 z)^{2}}, \\
& F_{3}(x, y, z)=\sqrt{4 x^{2}+(b+2 y)^{2}+(l+2 z)^{2}}, \\
& F_{4}(x, y, z)=\sqrt{4 x^{2}+(b-2 y)^{2}+(l+2 z)^{2}} .
\end{aligned}
$$

\subsection{Magnetic field of the ribbon busbar - visualization}

Distribution of the magnetic field modulus for the ribbon busduct of finite length on the plane $x O y$ is shown in the Figure 2, whereat this field is expressed in relative values as the function shown with the formula

$$
h^{(t)}(x, y, z)=\frac{H^{(t)}(x, y, z)}{H_{0}^{(t)}}
$$

where the magnetic field modulus [4]

$$
H^{(t)}(x, y, z)=\sqrt{\left[H_{x}^{(t)}(x, y, z)\right]^{2}+\left[H_{x}^{(t)}(x, y, z)\right]^{2}}
$$

and the reference magnetic field

$$
H_{0}^{(t)}=\frac{I}{2 b}
$$

Effect of the conductor length on the magnetic field distribution is shown [5] in the Figure 3. 


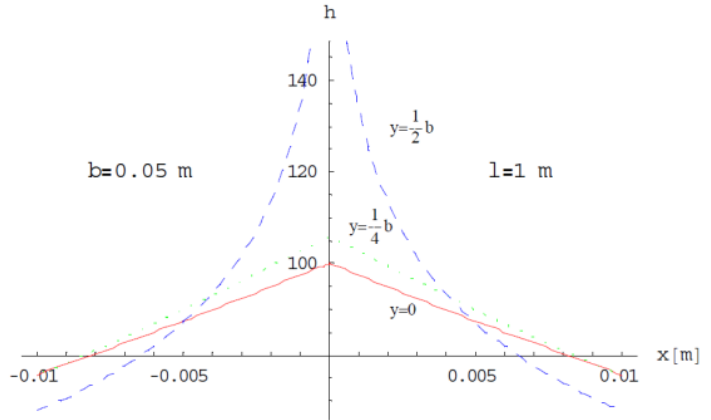

Fig. 2. Distribution of the magnetic field modulus on the plane $x O y$ of the ribbon busduct of finite length.

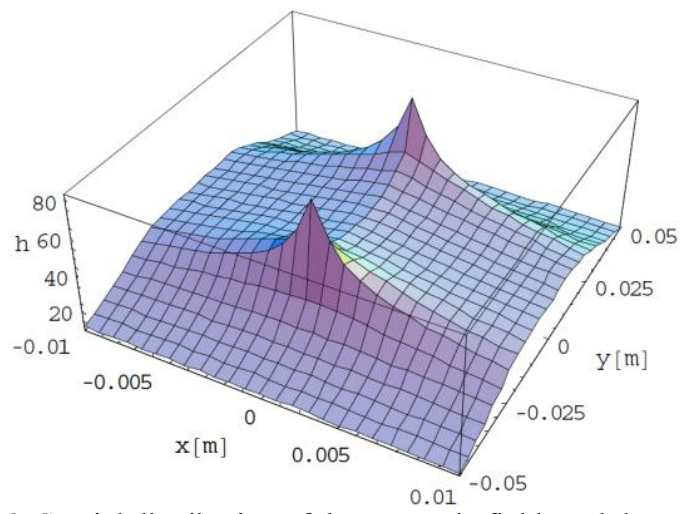

Fig. 3. Spatial distribution of the magnetic field modulus on the plane $z=l / 2$ of the ribbon busduct of finite length; $l=1 \mathrm{~m}, b=0.05 \mathrm{~m}$.

\section{Magnetic field line of the three ribbon busbar of finite length}

Our assumption is as follows: in the three-phase line of three ribbon busbars of dimensions $b \times l$ (Fig. 4) and the distance $d$ between them, there is the direct current or slow alternating sinusoidal current of complex rms values $\underline{I}_{1}, \underline{I}_{2}$ and $\underline{I}_{3}$.

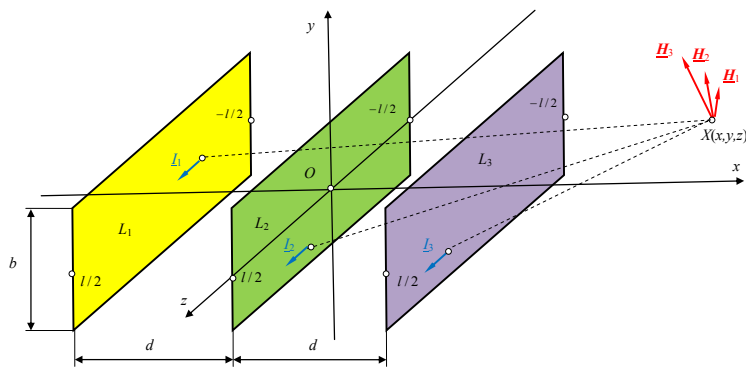

Fig. 4. The three-phase line of three ribbon conductors of finite length.

The method of magnetic field determination at any point $X(x, y, z)$ is the same as for the case of the threephase line of three rectangular busducts [1]. Distribution of the total magnetic field modulus on the plane $x O y$ for the three-phase line of three conductors in case of asymmetric currents in the busducts, i.e. $\underline{I}_{1}=\underline{I} \mathrm{e}^{\mathrm{j} 0^{\circ}}$, $\underline{I}_{2}=0.5 \underline{I} \mathrm{e}^{-\mathrm{j} 120^{\circ}}$ and $\underline{I}_{3}=-\underline{I}_{1}-\underline{I}_{2}=0.866 \underline{I} \mathrm{e}^{\mathrm{j} 150^{\circ}}$, is shown in the Figure 5, whereat this field is expressed in relative values as the function shown with the formula (3), where the reference magnetic field are expressed with the formula (5). Conductor length effect on distribution of the total magnetic field in such a line is shown in Figures 6.

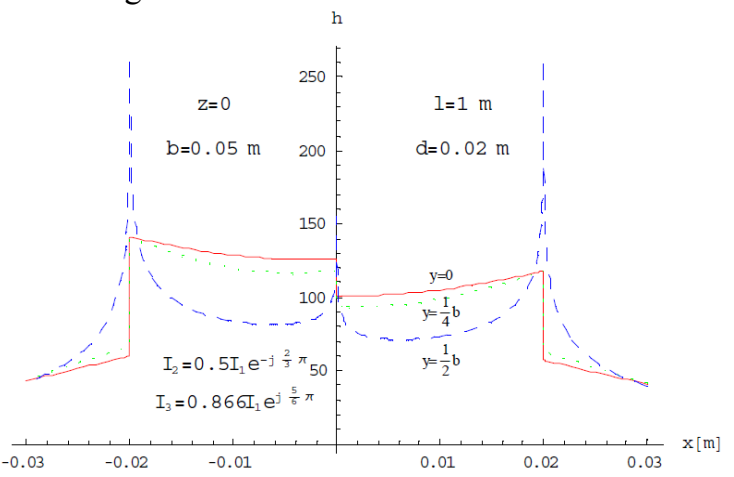

Fig. 5. Distribution of the magnetic field modulus of the threephase line of three ribbon busbars of finite length on the plane $x O y$ for asymmetric currents.

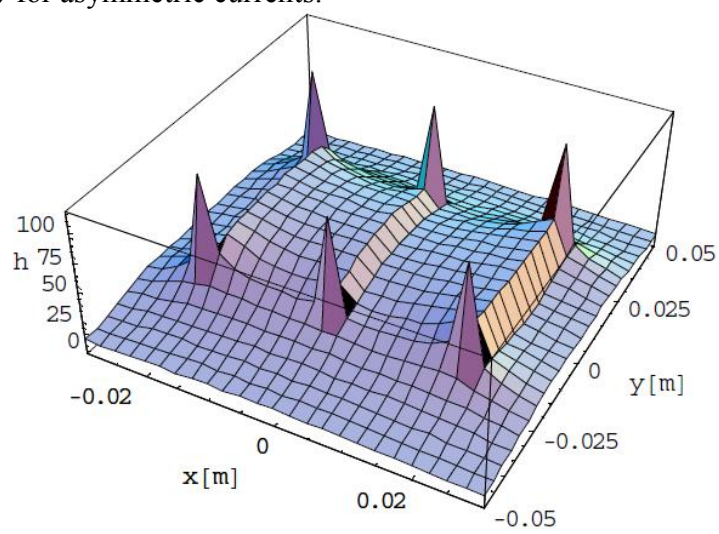

Fig. 6. Spatial distribution of the magnetic field modulus on the plane $z=l / 2$ for the three-phase line of three ribbon busbars of finite length for asymmetric currents; $l=1 \mathrm{~m}, d=0.02 \mathrm{~m}, b=$ $0.05 \mathrm{~m}$.

\section{Findings}

The derived formulas that describe the magnetic field have the complex structure even in the ribbon conductor and, based on them, it is difficult to foresee a change of the magnetic field distribution after change of, eg., this conductor length. However, using any program with a graphic module, eg. Mathematica, we have the ready to use, very good tool to visualize the obtained solutions quickly. As you can see in Figures 3 and 6, this allows quick field analysis after changes of geometrical or electrical parameters of systems under examination.

\section{References}

1. D. Kusiak, T. Szczegielniak, Electromagnetic calculations of busbars (in Polish), (Wyd. PCz, Czestochowa, 2017)

2. P. Sarajcev, R. Goic, Electr. Pow. Compo. Sys. 38, (2010)

3. Z. Piątek, B. Baron et. al., Prz. Elektrotech. 89/8, (2013)

4. Z. Piątek, Impedances of high-current busducts, (Wyd. PCz., Czestochowa, 2008)

5. H. Gliński, R. Grzymkowski et. al., Mathematica 8 (in Polish), (Wyd. Skalmierskiego, Gliwice, 2012) 